\title{
Optical Properties of Strained Wurtzite Gallium Phosphide Nanowires
}

\author{
J. Greil,$^{\dagger}$ S. Assali, ${ }^{\dagger}$ Y. Isono, ${ }^{\ddagger}$ A. Belabbes, ${ }^{\S, \|}$ F. Bechstedt, ${ }^{\S}$ F. O. Valega Mackenzie, ${ }^{\perp}$ A. Yu. Silov, ${ }^{\dagger}$ \\ E. P. A. M. Bakkers, ${ }^{\dagger, \#}$ and J. E. M. Haverkort* ${ }^{\dagger} \dagger$
}

${ }^{\dagger}$ Department of Applied Physics, Eindhoven University of Technology, 5600 MB, Eindhoven, The Netherlands

${ }^{\ddagger}$ Department of Mechanical Engineering, Kobe University, Kobe 657-8501, Japan

${ }^{\S}$ Institut für Festkörpertheorie und -optik, Friedrich-Schiller-Universität, Max-Wien-Platz 1, 07743 Jena, Germany

"King Abdullah University of Science \& Technology, Thuwal 23955-6900, Kingdom of Saudi Arabia

${ }^{\perp_{\text {TNO }}}$ Technical Sciences, De Rondom 1, 5612 AP, Eindhoven, The Netherlands

${ }^{\#}$ Kavli Institute of Nanoscience, Delft University of Technology, 2600 GA, Delft, The Netherlands

Supporting Information
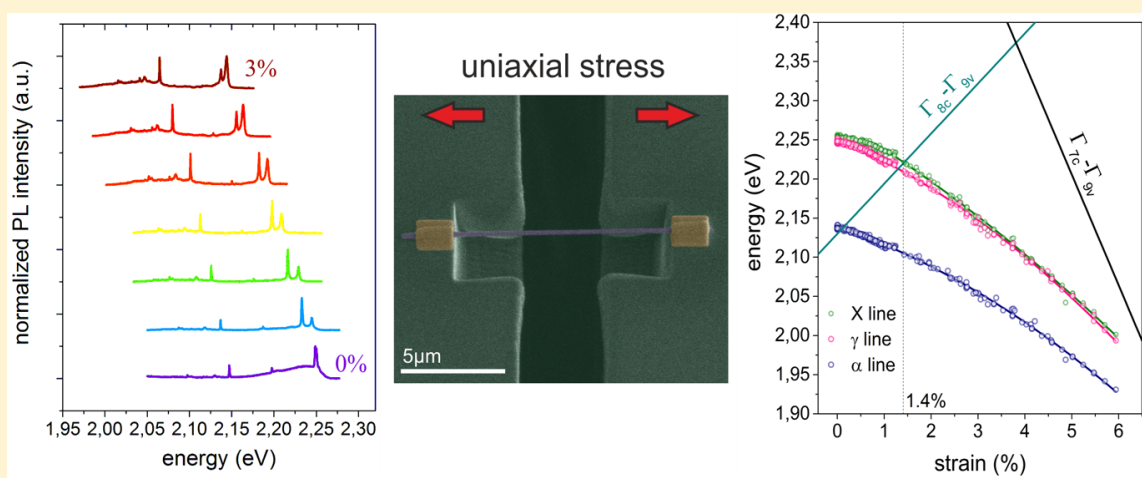

ABSTRACT: Wurtzite gallium phosphide (WZ GaP) has been predicted to exhibit a direct bandgap in the green spectral range. Optical transitions, however, are only weakly allowed by the symmetry of the bands. While efficient luminescence has been experimentally shown, the nature of the transitions is not yet clear. Here we apply tensile strain up to $6 \%$ and investigate the evolution of the photoluminescence (PL) spectrum of WZ GaP nanowires (NWs). The pressure and polarization dependence of the emission together with a theoretical analysis of strain effects is employed to establish the nature and symmetry of the transitions. We identify the emission lines to be related to localized states with significant admixture of $\Gamma_{7 \mathrm{c}}$ symmetry and not exclusively related to the $\Gamma_{8 \mathrm{c}}$ conduction band minimum (CBM). The results emphasize the importance of strongly bound staterelated emission in the pseudodirect semiconductor $\mathrm{WZ} \mathrm{GaP}$ and contribute significantly to the understanding of the optoelectronic properties of this novel material.

KEYWORDS: Wurtzite semiconductor, strain, localized state, photoluminescence, band structure, symmetry

allium phosphide has been predicted to exhibit a direct

bandgap in the wurtzite crystal structure. ${ }^{1-3}$ Bulk GaP, however, can only be grown in the zinc blende ( $\mathrm{ZB})$ crystal structure with an indirect bandgap. When dimensions are reduced to the geometry of a nanowire, the restriction to the $\mathrm{ZB}$ phase does not hold anymore and a high degree of control over the crystal phase can be achieved. ${ }^{4-7}$ Only recently, progress in synthesis techniques enabled the growth of phase pure WZ GaP nanowires. ${ }^{8,9}$ Thus, little experimental data on the band structure is available as of yet, ${ }^{8,10}$ and the main sources of information are theoretical studies. ${ }^{2,3,11}$ A pseudodirect bandgap is predicted with only weakly allowed or forbidden transitions from the lowest conduction band. ${ }^{11}$ DFT-LDA-1/2 simulations ${ }^{3,11}$ of the band structure of WZ $\mathrm{GaP}^{12}$ are shown in Figure 1a. The presented band structure includes quasiparticle corrections, which open the fundamental gap and interband energies, so that they can be directly (with an accuracy of about $0.1 \mathrm{eV}$ ) compared with experimental results. The underlying ground state calculations of strained and unstrained WZ GaP are performed using the density functional theory (DFT) as implemented in the Vienna $a b$ initio Simulation Package (VASP). ${ }^{13}$ On top of the resulting geometries the electronic bands are computed applying a recently developed approximate quasiparticle scheme, the socalled LDA-1/2 method, ${ }^{14,15}$ which, however, gives reasonable excitation energies and guarantees the correct description of the

Received: March 10, 2016

Revised: April 29, 2016

Published: May 13, 2016 

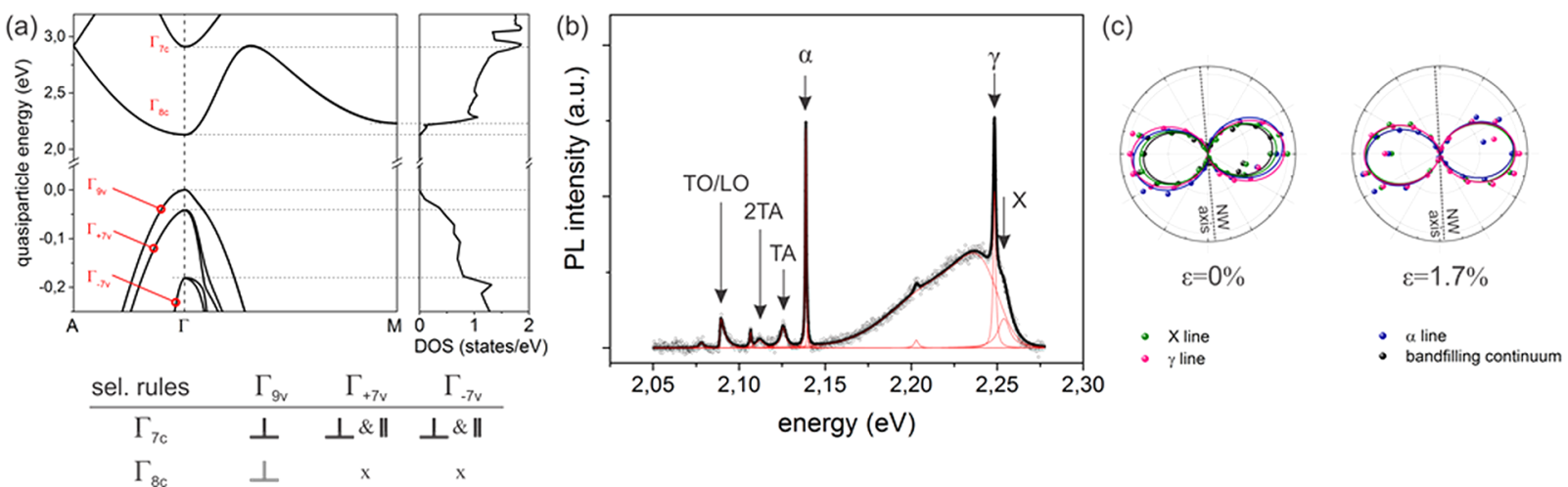

Figure 1. (a) Quasiparticle (QP) bandstructure, density of states (DOS), and optical selection rules of WZ GaP. Allowed transitions are indicated by their polarization with respect to the $c$-axis. Weak transitions are colored in gray and forbidden transitions are marked with an $X$. (b) PL emission spectrum of an unstrained WZ GaP NW at $4 \mathrm{~K}$. (c) Normalized polarization dependence of the NW integrated emission for the different features. All features are polarized perpendicular to the NW $c$-axis with a degree of polarization $>90 \%$ in all cases.

energy variations with the polytype. Details on the computational methods can be found in the Supporting Information. The simulations predict three nondegenerate valence bands, the heavy-hole $\left(\Gamma_{9 \mathrm{v}}\right)$, light-hole $\left(\Gamma_{7 \mathrm{v}+}\right)$, and split-off $\left(\Gamma_{7 \mathrm{v}-}\right)$ bands. The lowest conduction band $\Gamma_{8 c}$ is found at the $\Gamma_{\text {-point }}$ resulting in a direct bandgap of $2.13 \mathrm{eV}$. The $\Gamma_{8 \mathrm{c}}$ band is referred to as "dark" conduction band since optical transitions are only weakly allowed or fully forbidden by optical selection rules. Only for $k$-values with a finite wave vector outside the $\Gamma$ point, transition matrix elements are increasing. ${ }^{11}$ It should be emphasized, that other important III-V materials, such as WZ GaAs and WZ GaN, as well as II-VI materials like WZ ZnO and WZ CdS exhibit a direct bandgap with a "bright" lowest conduction band with $\Gamma_{7 \mathrm{c}}$ symmetry in the wurtzite crystal configuration.

Despite the pseudodirect bandgap, efficient light emission from WZ GaP NWs has been shown, ${ }^{8}$ but so far the nature of the transitions is not clearly established. The PL spectrum of an unstrained $\mathrm{WZ} \mathrm{GaP} \mathrm{NW}$ at high excitation density $(\sim 1 \mathrm{~kW} /$ $\left.\mathrm{cm}^{2}\right)$ and low temperature $(4 \mathrm{~K})$ is shown in Figure $1 \mathrm{~b}$. Typically two sharp lines are observed at 2.14 and $2.25 \mathrm{eV}$, labeled $\alpha$ and $\gamma$, respectively (keeping consistent nomenclature with ref 12 ). The width of these lines is remarkably narrow, less than $1 \mathrm{meV}$. The features at energies below the $\alpha$-line are identified as phonon replicas of the line and can be assigned to TA and TO/LO phonon modes based on the expected phonon energies from Raman characterization ${ }^{10}$ and applying folding arguments to the $\mathrm{ZB}$ values. ${ }^{16,17}$ The line labeled $\mathrm{X}$ is hidden as a shoulder in the unstrained NW spectrum but becomes more pronounced under strain. From temperature- and powerdependent characterization, the $\alpha, \gamma$, and $\mathrm{X}$ lines were assigned to excitonic recombination. ${ }^{12}$ The continuum-like emission in the range of $2.15-2.25 \mathrm{eV}$ strongly depends on the excitation power density and shows a clear drop at the $\gamma$-line. The continuum is attributed to bandfilling ${ }^{18}$ of the $\Gamma_{8 \mathrm{c}}$ band due to high excitation, with a shape determined by the k-dependence of the transition strength. ${ }^{11}$ The observation of bandfilling is consistent with the weakly allowed bandgap transition at $k=0$. Polarization-dependent characterization as shown in Figure 1c reveals strong linear polarization of the emission features $(\alpha, \gamma$, $\mathrm{X}$, and the continuum) perpendicular to the NW axis. The degree of polarization is higher than $90 \%$. The polarization dependence of the lines together with group-theoretical considerations for hexagonal crystals of the space group $\mathrm{C}_{6 v}{ }^{4}$ gives additional information on the states. Considering the polarization selection rules for states of $\Gamma_{7 \mathrm{c}}$ symmetry (Table in Figure 1a), any transition to $\Gamma_{-7 \mathrm{v}}$ or $\Gamma_{+7 \mathrm{v}}$ would lead to significant contributions of parallel polarized light. From the lack of any parallel component in the observed lines we conclude that only transitions to the heavy hole valence band $\Gamma_{9 \mathrm{v}}$ are involved. It is important to mention here that strain along the $c$-axis does not modify the symmetry properties of the crystal so the polarization selection rules do not change with strain. ${ }^{19}$ Thus, the direction and degree of polarization are independent of strain.

While bandfilling and phonon replicas are easily identified, determining the origin of the intriguingly sharp lines $\alpha$ and $\gamma$ is not straightforward. The pseudodirect band structure only very weakly allows for transitions at all; nevertheless, we observe a rich emission spectrum. Additionally the $\gamma$-line is found resonant in the continuum, which is an unexpected observation. An extensive study on the optical properties of unstrained WZ GaP NWs suggests that the $\alpha$ - and $\gamma$-lines can be assigned to strongly bound exciton transitions. ${ }^{12}$ To conclusively identify the emission lines, however, PL spectroscopy alone is not sufficient. Unambiguous identification of the nature and symmetry of the transitions requires pressure-dependent characterization. Photoluminescence characterization in combination with pressure studies proves a powerful tool to determine the energy band structure and band symmetries of new materials ${ }^{24-26}$ as well as the properties of yet unknown impurities. $^{27-29}$ Uniaxial strain can thereby provide more information than its hydrostatic counterpart but is often limited by sample fracture. ${ }^{30}$ For NWs it has been shown that the maximum permissible uniaxial strain approaches the theoretical maximum when the diameter is reduced, ${ }^{31,32}$ thus offering the opportunity to characterize a material at high strain levels. Several works have been published, analyzing the energy bandgap shift with strain in NWs. The apparent difficulties therein are, among others, to dynamically apply a controllable amount of stress in a defined direction and to characterize the material at low temperature. PL studies of NWs subject to static bending have been reported. ${ }^{33,34}$ The results are difficult to interpret, though, because of the superposed contributions of compressive and tensile strain in the bent regions. Signorello et al. have shown wide dynamic tunability of $\mathrm{ZB} \mathrm{GaAs} \mathrm{NWs}{ }^{35}$ and clarified the bandstructure of WZ GaAs $\mathrm{NWs}^{24}$ at $100 \mathrm{~K}$ and room temperature, respectively, by integration into a 3-point bending device. $\mathrm{WZ} \mathrm{ZnO} \mathrm{NWs}$ have been strained uniaxially and characterized in situ in an SEM chamber with 

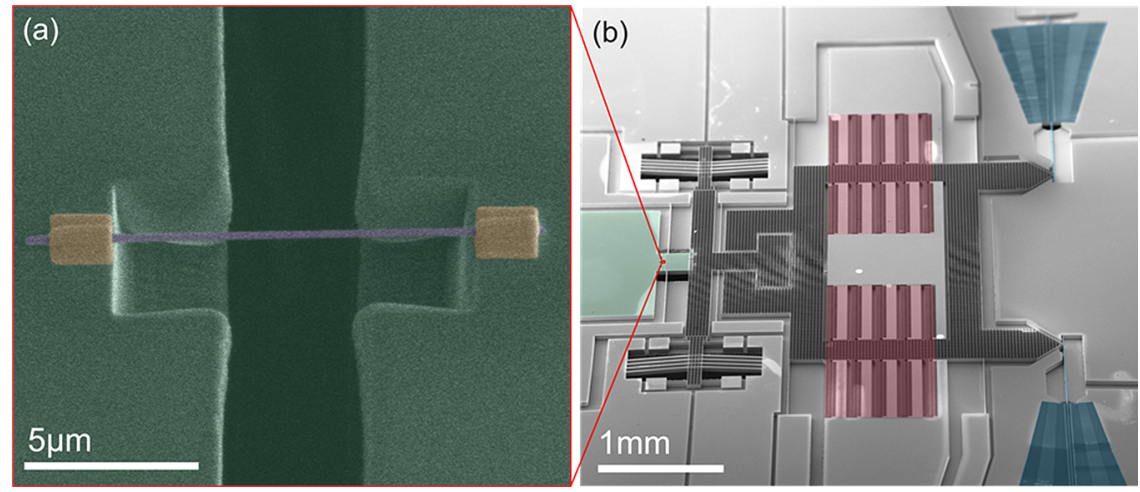

Figure 2. Single NW nanotensile testing device. Tilted view false-color SEM images (a) of a WZ GaP NW (blue) integrated into the device using electron beam induced deposition (gold) and (b) of the nanotensile testing device. ${ }^{20-23}$ The specimen area is colored green, the comb-drive array red, and the strain indicators light blue.
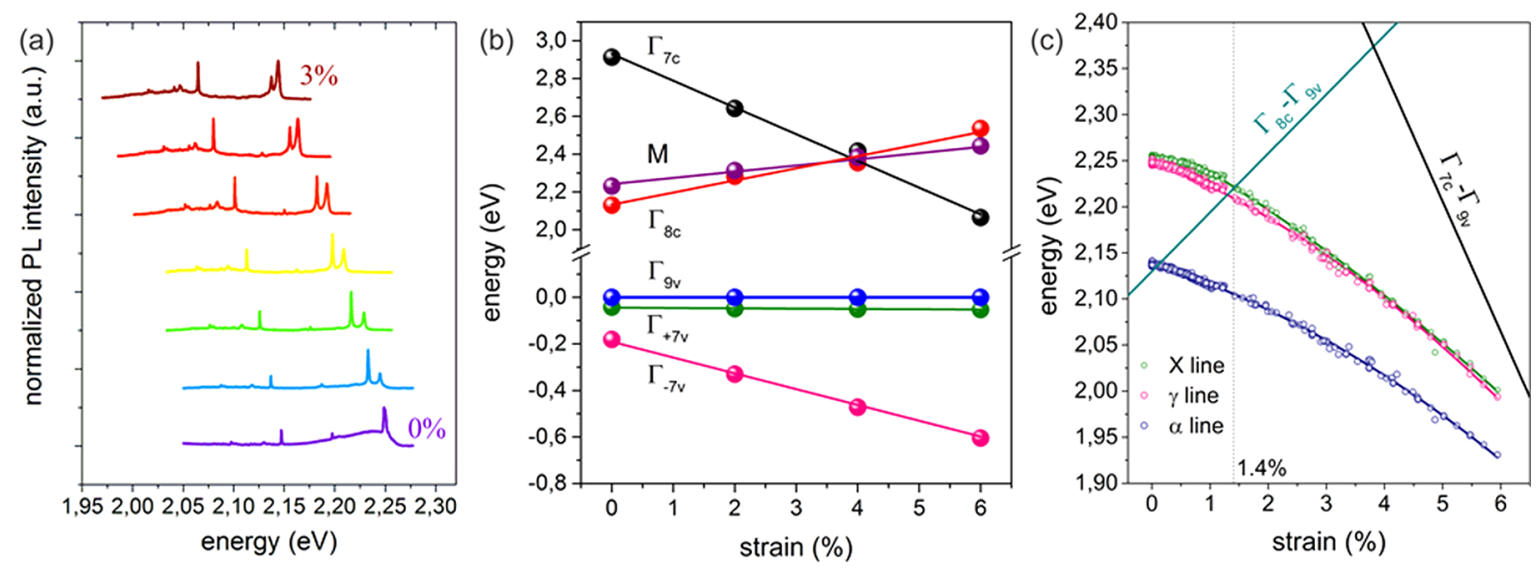

Figure 3. WZ GaP emission and band edge energies under uniaxial tensile strain. (a) Evolution of the emission spectrum with uniaxial tensile strain up to $3 \%$. (b) Results of $a b$ initio calculations for the QP energies of the valence- and conduction band states at the $\Gamma$ and $M$ points as a function of uniaxial tensile strain along the $c$-axis. The valence band maximum is used as the reference energy, thus the shift of $\Gamma_{9 \mathrm{v}}$ is zero. Solid lines represent linear fits to the data. The fitting parameters are listed in Table 2. (c) Transition energies extracted from $a b$ initio calculations in comparison with the experimental data. The experimental emission energies consistently red-shift with strain, while the fundamental $\Gamma_{8 \mathrm{c}}-\Gamma_{9 \mathrm{v}}$ bandgap is blue-shifting.

cathodoluminescence spectroscopy, however, with the restriction to room temperature. ${ }^{36}$ The findings reported for WZ-type $\mathrm{NWs}$ of GaAs, $\mathrm{ZnO}$, and $\mathrm{CdS}$ are in general consistent with theory, showing a decrease in bandgap-related PL energy with tensile uniaxial strain due to the red-shift of the CBM of $\Gamma_{7 \mathrm{c}}$ symmetry. In this work we employ high uniaxial tensile strain to determine the origin and symmetry properties of the transitions involved in $\mathrm{PL}$ at liquid helium temperature $(4 \mathrm{~K})$. The controlled application of strain to WZ GaP NWs is facilitated via integration into a nanotensile testing device ${ }^{20-23}$ (Figure 2). The peculiarity in interpretation of our data lies in the pseudodirect bandgap of $\mathrm{WZ} \mathrm{GaP}$. In contrast to the aforementioned WZ materials with a bright $\Gamma_{7 \mathrm{c}} \mathrm{CBM}, \mathrm{WZ}$ $\mathrm{GaP}$ exhibits a dark $\Gamma_{8 \mathrm{c}} \mathrm{CBM}$. By contrasting experimental PL data with theoretical predictions on the strain dependence of the band structure we provide a conclusive picture on the origin and symmetry of the transitions.

The strain dependence of the PL emission is shown in Figure 3a,c. Upon the application of uniaxial tensile strain, spectral features are consistently shifting toward lower energies. A remarkable maximum spectral shift of $250 \mathrm{meV}$ for all sharp lines was found at a strain level of $6 \%$. The shift of the peak positions as a function of strain is almost linear with a small quadratic correction. The coefficients of the fit to the emission line energies

$$
E=E_{0}+A \varepsilon+B \varepsilon^{2}
$$

can be found in Table 1 .

Table 1. Emission Line Energies and Strain Rates Obtained from PL Characterization:

\begin{tabular}{cccc} 
& $E_{0}(\mathrm{eV})$ & $A(\mathrm{eV} / \varepsilon)$ & $B\left(\mathrm{eV} / \varepsilon^{2}\right)$ \\
$\alpha$-line & 2.139 & -2.031 & -25.5 \\
$\gamma$-line & 2.251 & -2.511 & -30.8 \\
\hline
\end{tabular}

The spread of the emission energy is low, and no hysteretic behavior was found in several load cycles applied to the NW (see Supporting Information for details), indicating purely elastic deformation. Different diameters $(100$ and $220 \mathrm{~nm})$ as well as core-shell NWs $\left(d=250 \mathrm{~nm}, \mathrm{Al}_{40} \mathrm{Ga}_{60} \mathrm{P}\right.$ shell, $d_{\text {shell }} \approx$ $15 \mathrm{~nm}$ ) were characterized. All NWs show similar characteristic features and consistent behavior with strain, thus rendering dominant surface phenomena unlikely. The width and intensity of the lines as a function of strain typically show very distinct behavior for the $\alpha$-, $\gamma$-, and X-transition (see Supporting Information for details). With increasing strain, the $\gamma$-line that is initially dominating in unstrained NWs becomes less intense than the X-line (see Figure 3a). Also, a very rapid quench of the band filling continuum related to the fundamental $\Gamma_{8 \mathrm{c}}-\Gamma_{9 \mathrm{v}}$ gap 
is observed. The intensity of the $\alpha$-line, however, is only weakly affected by strain but saturates at higher excitation densities. Though in most of the wires we find all the spectral features, few single wires exhibit either the $\alpha$-line or the $\gamma-\mathrm{X}$-lines only. The independent observation of $\alpha$ - and $\gamma-\mathrm{X}$-lines, the saturation of the $\alpha$-line with excitation power, and the quench of the $\gamma$-line with strain suggests a different origin for the $\alpha$-line and the $\gamma$-X-lines. The shift of the TO/LO phonon energy, and thus the relative shift of the phonon replica with respect to the $\alpha$-line position, is found to depend linearly on strain in the $\mathrm{NW}$, indicating pure elastic deformation ${ }^{24}$ (see Supporting Information for details).

To establish the symmetry properties of the optical transitions in $\mathrm{WZ} \mathrm{GaP}$ based on pressure experiments, the strain dependence of the different band edges needs to first be known. The strain dependence of the band edge energies was theoretically evaluated at the $\Gamma$ and $M$ point in the Brillouin zone (Figure $3 \mathrm{~b}$ ). An increase of the fundamental band gap as well as an increase of the energy for the transitions to the light hole and split-off band can be seen. Only the "bright" conduction band $\Gamma_{7 c}$ is found to decrease in energy with strain. The quasiparticle energy $E_{i}$ can be fitted according to

$$
E_{i}=E_{0}+d_{\mathrm{u}} \varepsilon
$$

with $E_{0}$ the zero strain energy, $d_{\mathrm{u}}$ the uniaxial strain coefficient, and $\varepsilon$ the uniaxial strain.

The energetic position and strain coefficients of the band edges can be found in Table 2 .

Table 2. Band Edge Energies and Strain Rates Obtained from $a b$ Initio Calculations

\begin{tabular}{lcccccl} 
& $\Gamma_{7 \mathrm{c}}$ & $\Gamma_{8 \mathrm{c}}$ & $\mathrm{M}$ & $\Gamma_{9 \mathrm{v}}$ & $\Gamma_{+7 \mathrm{v}}$ & $\Gamma_{-7 \mathrm{v}}$ \\
$E_{0}(\mathrm{eV})$ & 2.91 & 2.13 & 2.23 & 0 & -0.04 & -0.18 \\
$d_{\mathrm{u}}(\mathrm{eV} / \varepsilon)$ & -14.1 & 6.4 & 3.3 & 0 & -0.1 & -6.8 \\
\hline
\end{tabular}

In Figure $3 c$ the transition energies from the conduction bands to $\Gamma_{9 \mathrm{v}}$ as determined by ab initio calculations as well as the experimentally found emission energies are plotted as a function of strain. The calculated value for the fundamental bandgap of $2.13 \mathrm{eV}$ at zero strain is in good agreement with the $2.19 \mathrm{eV}$ that have been determined experimentally in ref 12 . At zero strain, the $\alpha$-exciton is thus bound $50 \mathrm{meV}$ deep in the
$\Gamma_{8 \mathrm{c}}-\Gamma_{9 \mathrm{v}}$ bandgap, while the $\gamma$-exciton is found resonant in the conduction band. A low density of states (DOS) in the lowest conduction band valley has been reported to promote the existence of resonant states in the conduction band. ${ }^{37,38}$ Our calculations confirm the low DOS at $\Gamma$ (Figure 1a), thus explaining the occurrence of a resonant state. For the interaction of a discrete level and a continuum, theoretical considerations suggest a Fano line shape for the emission. ${ }^{38}$ The resonant state is expected to modify the continuum line shape and redistribute oscillator strength, so that broad enhancement below the resonance and a dip at the highenergy side is produced. There is, however, experimental data showing emission from resonant states in a symmetric, nonFano line shape. ${ }^{37}$ For the $\gamma$-line, we find a good fit with a Lorentzian. The $k$-dependence of the underlying continuum, though, adds some uncertainty in the exact line shape.

Under tensile strain, the calculated fundamental $\Gamma_{8 \mathrm{c}}-\Gamma_{9 \mathrm{v}}$ bandgap transition responsible for bandfilling is strongly blue shifting. This is consistent with the observed rapid decrease of the bandfilling continuum intensity with strain (see Supporting Information for details). We observe a strong cutoff of the continuum at the $\gamma$-line that is probably induced by depopulation of the continuum in favor of the $\gamma$-line. Thus, a blue-shift decreases the occupation of the continuum leading to the decrease in intensity. By comparing the experimentally observed shift for the $\alpha, \gamma$, and $\mathrm{X}$ exciton lines under strain with the theoretically calculated shift of the conduction and valence band edges, it is clear that the $\alpha, \gamma$, and $\mathrm{X}$ emission lines do not follow the fundamental $\Gamma_{8 \mathrm{c}}-\Gamma_{9 \mathrm{v}}$ bandgap transition as would be expected for free or shallow bound excitons. Instead, all the lines exhibit similar negative slopes intermediate between the $\Gamma_{8 \mathrm{c}}$ and the $\Gamma_{7 \mathrm{c}}$, indicating emission related to states of mixed symmetry. Localized states are known to introduce this mixing. The wave function of shallow defects extends over many unit cells and can be described by the effective mass theory, whereas deep centers exhibit highly localized wave functions. The localization in real space translates to a strong delocalization in $k$-space and the wave function of deep centers in general involves Bloch functions from several bands. ${ }^{39,40}$ Thus, free excitons and excitons bound to shallow states follow the bandgap, whereas localized states shift according to a superposition of all minima in the band $d^{41,42}$ as observed in our experiment. To evaluate the symmetry properties of a
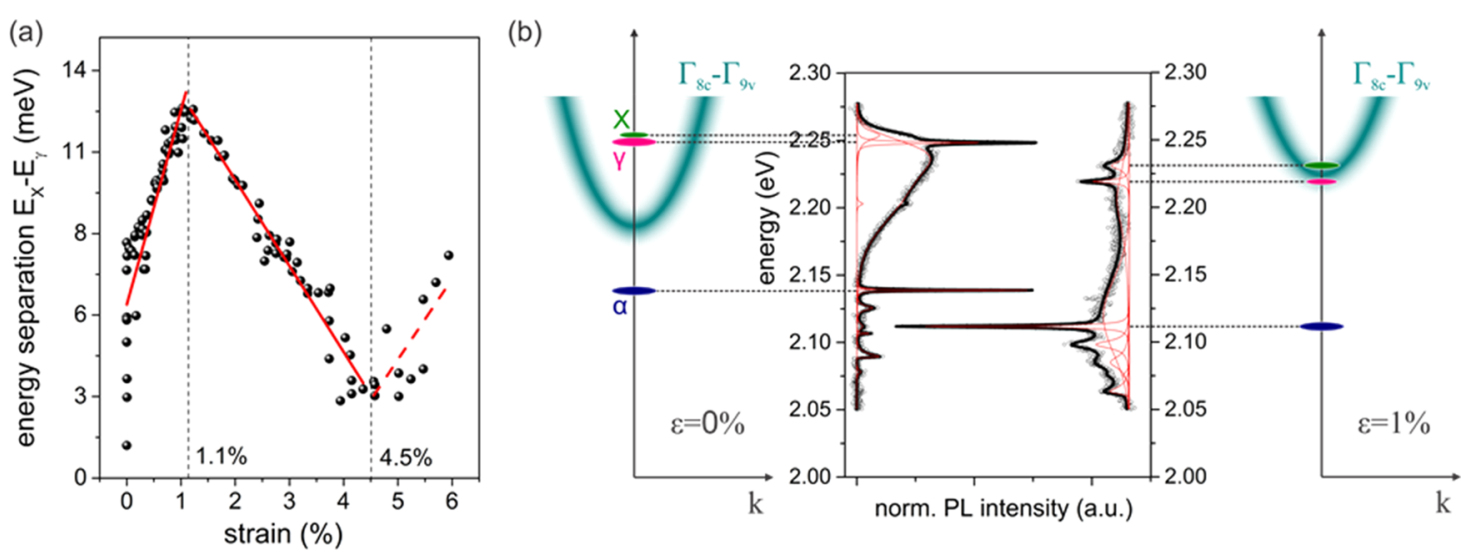

Figure 4. $\gamma-\mathrm{X}$ splitting and proposed alignment of the localized states. (a) Strain dependence of the energy separation between the X-line and the $\gamma$ line $\left(E_{\mathrm{X}}-E_{\gamma}\right)$. (b) PL spectra at $0 \%$ and $1 \%$ strain along with sketches of the proposed relative alignment of states. In unstrained WZ GaP the state related to the $\alpha$-transition is bound $50 \mathrm{meV}$ within the bandgap, while the states related to the $\gamma$ - and X-transitions are resonant within the conduction band. 

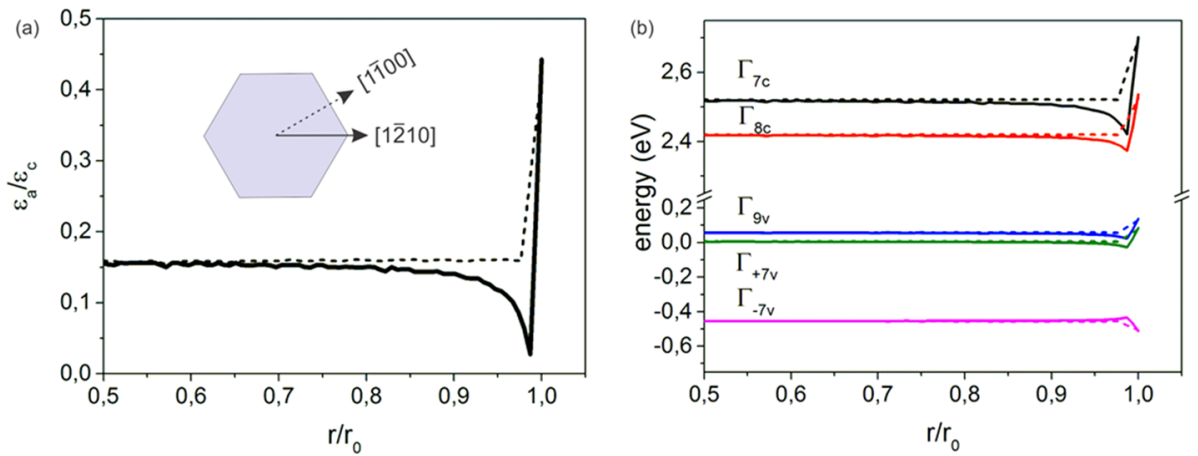

Figure 5. Radial strain distribution and band edge energies. (a) The radial in-plane strain distribution of a $115 \mathrm{~nm}$ diameter NW is calculated, and the ratio of in-plane to c-axial strain (i.e., the Poisson ratio $\nu=\varepsilon_{\mathrm{a}} / \varepsilon_{\mathrm{c}}$ ) is given. Strain was evaluated along the two crystal directions indicated by arrows in the sketch. The in-plane strain is deviating from bulk-like behavior only in the outermost $10 \%$ of the NW radius and in the direction toward the edge. (b) The band edge energies at a uniaxial strain of $4 \%$ are calculated according to the strain distribution in panel a. A red-shift of a few tens of $\mathrm{meV}$ in the edge region is expected.

highly localized state, calculations were exemplarily performed for the nitrogen impurity in WZ GaP. GaP doped with $\mathrm{N}$ atoms is modeled with a 108 atom wurtzite supercell where one $\mathrm{P}$ site is substituted by $\mathrm{N}$. This corresponds to a nominal $\mathrm{N}$ doping concentration of $1.8 \%$ (see Supporting Information for details). At this nitrogen concentration we find an impurity-related $\mathrm{CBM}$ of $\Gamma_{7 \mathrm{c}}$ symmetry. The transition strength from the new band to the valence band maximum increases by a factor of more than 20 when compared to the pure WZ GaP bandgap transition. Thus, the perturbation due to a deep center, e.g., nitrogen, may introduce a highly efficient transition to the $\Gamma_{9 \mathrm{v}}$ valence band by mixing with other states, e.g., $\Gamma_{70}$ due to local symmetry lowering. Based on these theoretical considerations and the strain dependence we assign the $\alpha$-, $\gamma$-, and Xtransitions to highly localized states with significant admixture of $\Gamma_{7 \mathrm{c}}$ symmetry. Possible impurities that could introduce these states in WZ GaP along with their concentration based on atom probe tomography analysis are listed in ref 12 . Carbon and oxygen impurities were found on the order of $5 \times 10^{17} \mathrm{~cm}^{-3}$. The identification of the exact species goes beyond the scope of this work, though.

We now focus on another intriguing feature of the strain dependence, that is the splitting of the $\gamma$ - and X-lines with strain as shown in Figure 4a. A pronounced nonmonotonic relative shift of the $\gamma$-line and the close-by X-line with strain is observed. The splitting energy between the two lines increases linearly, followed by a linear decrease with $\sim 1 / 2$ the slope of the increase. For all devices, the splitting exhibits a remarkably sharp and consistent turning point at $\sim 1 \%$ tensile strain. At $4.5 \%$ the energy separation apparently increases again, even though it is not unambiguously possible to determine whether the lines are crossing or anticrossing from the weak emission at high strain levels. It can be seen in Figure $3 \mathrm{c}$ that at $\sim 1.4 \%$ strain the $\gamma$ - and X-lines are crossing the predicted $\Gamma_{8 \mathrm{c}}-\Gamma_{9 \mathrm{v}}$ transition energy. The crossing occurs close (within the simulation uncertainty) to the maximum of the $\gamma$-X splitting at $\sim 1 \%$ strain, and the abrupt change in the slope of the $\gamma-\mathrm{X}$ splitting might be caused by repulsion between the X-state and the band edge close to the crossing point. The phenomenon of level repulsion between a localized state and the band edge has been shown to account for the changes in bandstructure observed in pressure experiments on nitrogen-doped III-V materials. ${ }^{43}$ With increasing strain, the $\mathrm{X}$-line is pushed away from the $\Gamma_{8 c}$ band edge, while the $\gamma$-line seems unaffected, thus increasing the $\gamma-\mathrm{X}$ energy separation until the crossing point is reached close to $1 \%$ strain. Beyond $1 \%$, repulsion of the X-line from the band edge again decreases the $\gamma-X$ splitting. In Figure $4 \mathrm{~b}$ we show the proposed relative alignment of states and the corresponding PL spectra for $0 \%$ and close to the crossing point at $1 \%$ strain. The zero strain $\Gamma_{8 \mathrm{c}}-\Gamma_{9 \mathrm{v}}$ band gap is set at $2.19 \mathrm{eV}$ according to the experimental findings of ref 12 . Considering the theoretically derived strain rates given in Table 2 the crossing point is found at $1 \%$, consistent with the proposed mechanism.

In order to be conclusive, we like to exclude other mechanisms that might introduce a red-shift in the excitonic emission. Since we are applying strain to nanoscale objects and not bulk samples, surface effects may become more prominent $^{36,44,45}$ and the strain distribution within the NW has to be considered. Furthermore, an electric field due to the piezoelectric effect potentially leads to a Stark-shift of the emission.

To study the effects of strain distribution in the nanowires, molecular statics calculations have been performed. For this, atomistic representations of hexagonal NWs with different diameter were built. Each NW was modeled completely in the direction perpendicular to the longitudinal axis with free boundaries to allow for surface relaxation. However, periodic boundary conditions were imposed in the longitudinal direction after 5 unit cells to simulate infinitely long wires. Interaction between atomic pairs was described using the gallium phosphide potential as developed previously in ref 46 and using the LAMMPS simulation engine. ${ }^{47}$ At first the equilibrium configuration of the nanowires was found by minimizing their total energy while also allowing for relaxation of the main axis. Afterward, the nanowires were strained uniformly in the longitudinal direction. Minimization was performed again, but this time the main axis was not allowed to change in order to maintain the imposed strain. This results in relaxation of the NW surfaces and the entire plane perpendicular to the main axis. Figure 5a shows the radial inplane strain distribution as obtained from the calculations. Inplane strain is derived from radial atomic displacement along the two directions indicated. The radial distribution is homogeneous throughout the NW. In the last few surface layers, however, in-plane strain is relaxed due to atomic rearrangement. There is a remarkable difference of strain distribution along the radial direction toward the facet and toward the edges. Strain in the direction toward the facets is constant bulk-like and only increases in the outermost atomic 
layer. Strain in the direction toward the edges, though, is decreasing significantly in the outer $10 \%$ of the radius and only at the outermost layer increasing again. Using the strain distribution as an input parameter for the band structure calculations, a radial profile of the band edges can be obtained. It is found that the amount of band gap lowering at the edges is only on the order of tens of $\mathrm{meV}$ at $4 \%$ strain and is thus not capable to account for the observed red-shift in Figure 3c. Additionally the volume close to the edges with lowered bandgap is small compared to the volume of the NW. Hence we expect the strain inhomogeneity not to significantly contribute to the energy shift of the lines. This is in contrast to the findings from ref 36 on $\mathrm{WZ} \mathrm{ZnO}$ NWs. Therein a sizedependent bandgap modulation under strain was found with a competition between core-dominated and shell-dominated contributions. Strong surface relaxation effects were proposed to induce a surface shrinking strain and thus a diameter dependence. The effect might be a specific feature of the surface reconstruction of $\mathrm{WZ} \mathrm{ZnO}$, though. In $\mathrm{WZ} \mathrm{GaP}$ we find no experimental evidence of such strong influence of the surface on the optical properties since the PL spectrum and strain dependence of the transitions is not altered by the NW diameter.

On the other hand, WZ GaP is a pyro- and piezoelectric material, i.e., strain might induce internal electric fields in addition to the spontaneous polarization field. ${ }^{4,49}$ These fields can shift transition energies via the Stark effect. ${ }^{50,51}$ There are several arguments, though, rendering it unlikely that a piezoelectric field induces the observed red-shift in the excitonic emission. First, the polarization field will be screened by free photoexcited carriers and surface charges. ${ }^{52,53}$ Surface charges may arise from the polarization discontinuity at the surface, ${ }^{52}$ adsorbates, ${ }^{54}$ or trap states. ${ }^{55}$ From excitation powerdependent characterization we do not observe a blue-shift of the emission with increasing power, as would be characteristic of Stark-shifted states. ${ }^{56}$ As a further limit, polarization fields can only be maintained up to a critical distance $d_{\text {crit }}$ that is given by the band gap $E_{\mathrm{g}}$ and the polarization field $\xi, d_{\text {crit }}=E_{\mathrm{g}} / \xi^{53}$ Over the length and width of a NW (typically $10 \mu \mathrm{m}$ and 200 $\mathrm{nm}$ ), a field of only $\sim 2$ and $\sim 100 \mathrm{kV} / \mathrm{cm}$ can be maintained, respectively. The shift of exciton emission due to electric fields is furthermore associated with severe broadening of the emission due to field ionization. The broadening limits the shift to $\sim 10 \%$ of the exciton binding energy. ${ }^{57}$ To determine the effect of an electric field on the excitonic emission experimentally, bias was applied along a NW (see Supporting Information for details). Quenching and significant broadening of the $\gamma$-line similar to the strain-induced effect was indeed found for an electric field on the order of $100 \mathrm{kV} / \mathrm{cm}$. The width and intensity of the $\alpha$-line, however, were only weakly affected by the electric field, indicating a larger binding energy and/or higher degree of localization of the $\alpha$-compared to the $\gamma$-exciton. The shift of the emission energy for both lines, though, was only on the order of single $\mathrm{meV}$ at a field of 100 $\mathrm{kV} / \mathrm{cm}$. These results render the piezoelectric and Stark effect very unlikely to contribute to the observed red-shift. A small unscreened portion of the piezoelectric field, though, might well account for the broadening observed in the $\gamma$-line with strain.

Summarizing, photoluminescence emission from phase-pure $\mathrm{WZ} \mathrm{GaP}$ NWs was analyzed as a function of uniaxial tensile strain to gain further insight into the nature of the transitions. Despite the pseudodirect nature of $\mathrm{WZ} \mathrm{GaP}$, excitonic lines dominate the PL spectrum. Based on the pressure dependence and $a b$ initio calculations of the bandstructure we propose the emission lines to be related to localized states. The symmetry of the states is determined by significant admixture of $\Gamma_{7 \mathrm{c}}$ and from the polarization selection rules, we identify transitions to the $\Gamma_{9 \mathrm{v}}$ heavy hole band. The proposed mechanism is further justified by calculations revealing the mixing of states due to local symmetry lowering induced by a deep center. Other mechanisms that could potentially shift the emission with respect to the theoretically predicted band gap energy are discussed and excluded.

\section{ASSOCIATED CONTENT}

\section{Supporting Information}

The Supporting Information is available free of charge on the ACS Publications website at DOI: 10.1021/acs.nanolett.6b01038.

Additional figures and information (PDF)

\section{AUTHOR INFORMATION}

\section{Corresponding Author}

*E-mail: J.E.M.Haverkort@tue.nl.

\section{Present Addresses}

(J.G.) Vienna University of Technology, Institute of Solid State Electronics, Floragasse 7, 1040 Vienna, Austria.

(S.A.) Department of Engineering Physics, Polytechnique Montréal, C.P. 6079, Succ. Centre-Ville, Montréal, Québec H3C 3A7, Canada.

\section{Notes}

The authors declare no competing financial interest.

\section{ACKNOWLEDGMENTS}

J.G. acknowledges funding by the Austrian Science Fund (FWF): Proj. No. J3540-N30. This work was supported by the Dutch Organization for Scientific Research (NWO-VICI 700.10.441).

\section{REFERENCES}

(1) Yeh, C.-Y.; Wei, S.-H.; Zunger, A. Phys. Rev. B: Condens. Matter Mater. Phys. 1994, 50 (4), 2715-2718.

(2) De, A.; Pryor, C. E. Phys. Rev. B: Condens. Matter Mater. Phys. 2010, 81, 155210.

(3) Belabbes, A.; Panse, C.; Furthmüller, J.; Bechstedt, F. Phys. Rev. B: Condens. Matter Mater. Phys. 2012, 86, 075208.

(4) Joyce, H. J.; Wong-Leung, J.; Gao, Q.; Tan, H. H.; Jagadish, C. Nano Lett. 2010, 10, 908-915.

(5) Glas, F.; Harmand, J.-C.; Patriarche, G. Phys. Rev. Lett. 2007, 99, 146101.

(6) Dick, K. A.; Caroff, P.; Bolinsson, J.; Messing, M. E.; Johansson, J.; Deppert, K.; Wallenberg, L. R.; Samuelson, L. Semicond. Sci. Technol. 2010, 25, 024009.

(7) Shtrikman, H.; Popovitz-Biro, R.; Kretinin, A.; Houben, L.; Heiblum, M.; Bukała, M.; Galicka, M.; Buczko, R.; Kacman, P. Nano Lett. 2009, 9 (4), 1506-1510.

(8) Assali, S.; Zardo, I.; Plissard, S.; Kriegner, D.; Verheijen, M. A.; Bauer, G.; Meijerink, A.; Belabbes, A.; Bechstedt, F.; Haverkort, J. E. M.; Bakkers, E. P. A. M. Nano Lett. 2013, 13, 1559-1563.

(9) Husanu, E.; Ercolani, D.; Gemmi, M.; Sorba, L. Nanotechnology 2014, 25, 205601.

(10) Panda, J. K.; Roy, A.; Gemmi, M.; Husanu, E.; Li, A.; Ercolani, D.; Sorba, L. Appl. Phys. Lett. 2013, 103, 023108.

(11) Bechstedt, F.; Belabbes, A. J. Phys.: Condens. Matter 2013, 25, 273201. 
(12) Assali, S.; Greil, J.; Zardo, I.; Belabbes, A.; de Moor, M. W. A.; Kölling, S.; Koenraad, P. M.; Bechstedt, F.; Bakkers, E. P. A. M.; Haverkort, J. E. M. Under review, 2016.

(13) Kresse, G.; Furthmüller, J. Phys. Rev. B: Condens. Matter Mater. Phys. 1996, 54, 11169.

(14) Ferreira, L. G.; Marques, M.; Teles, L. K. Phys. Rev. B: Condens. Matter Mater. Phys. 2008, 78, 125116.

(15) Ferreira, L. G.; Marques, M.; Teles, L. K. AIP Adv. 2011, 1, 032119.

(16) Dean, P. J.; Thomas, D. G. Phys. Rev. 1966, 150 (2), 690.

(17) Pödör, B. Phys. Status Solidi B 1983, 120, 207-213.

(18) Burstein, E. Phys. Rev. 1954, 93, 632-633.

(19) Tronc, P.; Kitaev, Y. E.; Wang, G.; Limonov, M. F. Phys. Status Solidi B 1998, 210, 471-474.

(20) Oh, H.-J.; Omori, H.; Sadakata, M.; Tsubokura, I.; Isono, Y. J. Microelectromech. Syst. 2014, 23 (4), 944-954.

(21) Kiuchi, M.; Matsui, S.; Isono, Y. J. Micromech. Microeng. 2008, 18 (6), 065011.

(22) Kiuchi, M.; Matsui, S.; Isono, Y. J. Microelectromech. Syst. 2007, 16 (2), 191-201.

(23) Oh, H.; Kawase, S.; Hanasaki, I.; Isono, Y. Jpn. J. Appl. Phys. 2014, 53, 027201.

(24) Signorello, G.; Lörtscher, E.; Khomyakov, P. A.; Karg, S.; Dheeraj, D. L.; Gotsmann, B.; Weman, H.; Riel, H. Nat. Commun. 2014, 5, 3655.

(25) Wagner, M. R.; Callsen, G.; Reparaz, J. S.; Kirste, R.; Hoffmann, A.; Rodina, A. V.; Schleife, A.; Bechstedt, F.; Phillips, M. R. Phys. Rev. B: Condens. Matter Mater. Phys. 2013, 88, 235210.

(26) Shan, W.; Hauenstein, R. J.; Fischer, A. J.; Song, J. J.; Perry, W. G.; Bremser, M. D.; Davis, R. F.; Goldenberg, B. Phys. Rev. B: Condens. Matter Mater. Phys. 1996, 54 (19), 13460-13463.

(27) Mooney, P. M. J. Appl. Phys. 1990, 67 (3), R1.

(28) Chen, S. J.; Liu, Y. C.; Shao, C. L.; Xu, C. S.; Liu, Y. X.; Liu, C. Y.; Zhang, B. P.; Wang, L.; Liu, B. B.; Zou, G. T. Appl. Phys. Lett. 2006, 88 (13), 133127.

(29) Ren, S. Y.; Dow, J. D.; Wolford, D. J. Phys. Rev. B: Condens. Matter Mater. Phys. 1982, 25 (12), 7661-7665.

(30) Cardona, M. Phys. Status Solidi B 1996, 198, 5-21.

(31) Smith, D. A.; Holmberg, V. C.; Korgel, B. A. ACS Nano 2010, 4 (4), 2356-2362.

(32) Zhu, Y.; Xu, F.; Qin, Q.; Fung, W. Y.; Lu, W. Nano Lett. 2009, 9 (11), 3934-3939.

(33) Han, X.; Kou, L.; Lang, X.; Xia, J.; Wang, N.; Qin, R.; Lu, J.; Xu, J.; Liao, Z.; Zhang, X.; Shan, X.; Song, X.; Gao, J.; Guo, W.; Yu, D. Adv. Mater. 2009, 21 (48), 4937-4941.

(34) Sun, L.; Kim, D. H.; Oh, K. H.; Agarwal, R. Nano Lett. 2013, 13 (8), 3836-3842.

(35) Signorello, G.; Karg, S.; Björk, M. T.; Gotsmann, B.; Riel, H. Nano Lett. 2013, 13 (3), 917-924.

(36) Wei, B.; Zheng, K.; Ji, Y.; Zhang, Y.; Zhang, Z.; Han, X. Nano Lett. 2012, 12 (9), 4595-4599.

(37) Scifres, D. R.; Macksey, H. M.; Holonyak, N. J.; Dupuis, R. D.; Zack, G. W.; Duke, C. B.; Kleiman, G. G.; Kunz, A. B. Phys. Rev. B 1972, 5 (6), 2206-2215.

(38) Altarelli, M. Phys. Rev. B 1975, 11 (12), 5031.

(39) Bassani, F.; Iadonisi, G.; Preziosi, B. Rep. Prog. Phys. 1974, 37 (9), 1099-1210.

(40) Yu, P. Y.; Cardona, M. Fundamentals of Semiconductors; Springer-Verlag: Berlin Heidelberg, 2010.

(41) Chadi, D. J.; Chang, K. J. Phys. Rev. Lett. 1988, 61 (7), 873-876.

(42) Wetzel, C.; Suski, T.; Ager, J. W., III; Weber, E. R.; Haller, E. E.; Fischer, S.; Meyer, B. K.; Molnar, R. J.; Perlin, P. Phys. Rev. Lett. 1997, 78 (20), 3923-3926.

(43) Klar, P. J.; Teubert, J.; Güngerich, M.; Niebling, T.; Grüning, H.; Heimbrodt, W.; Volz, K.; Stolz, W.; Polimeni, A.; Capizzi, M.; O’Reilly, E. P.; Lindsay, A.; Galluppi, M.; Geelhaar, L.; Riechert, H.; Tomić, S. Phys. Status Solidi B 2007, 244 (1), 24-31.

(44) Miller, R. E.; Shenoy, V. B. Nanotechnology 2000, 11, 139-147.
(45) Agrawal, R.; Peng, B.; Gdoutos, E. E.; Espinosa, H. D. Nano Lett. 2008, 8 (11), 3668-3674.

(46) Ribeiro-Silva, C. I.; Rino, J. P.; Gonçalves, L. G. V.; Picinin, A. J. Phys.: Condens. Matter 2011, 23 (5), 055801.

(47) Plimpton, S. J. Comput. Phys. 1995, 117 (1), 1-19.

(48) Al-Zahrani, H. Y. S.; Pal, J.; Migliorato, M. A.; Tse, G.; Yu, D. Nano Energy 2015, 14, 382-391.

(49) Kriegner, D.; Assali, S.; Belabbes, A.; Etzelstorfer, T.; Holý, V.; Schülli, T.; Bechstedt, F.; Bakkers, E. P. A. M.; Bauer, G.; Stangl, J. Phys. Rev. B: Condens. Matter Mater. Phys. 2013, 88, 115315.

(50) Blossey, D. F. Phys. Rev. B 1970, 2 (10), 3976-3990.

(51) Li, D.; Zhang, J.; Zhang, Q.; Xiong, Q. Nano Lett. 2012, 12, 2993-2999.

(52) Mayrock, O.; Wünsche, H.-J.; Henneberger, F. Phys. Rev. B: Condens. Matter Mater. Phys. 2000, 62 (24), 16870-16880.

(53) Wood, C., Jena, D., Eds. Polarization Effects in Semiconductors: From Ab Initio Theory to Device Applications; Springer: New York, 2008.

(54) Mönch, W. J. Vac. Sci. Technol., B: Microelectron. Process. Phenom. 1986, 4 (4), 1085.

(55) Norman, D.; McGovern, I. T.; Norris, C. Phys. Lett. A 1977, 63 (3), 384-386.

(56) Lähnemann, J.; Brandt, O.; Pfüller, C.; Flissikowski, T.; Jahn, U.; Luna, E.; Hanke, M.; Knelangen, M.; Trampert, A.; Grahn, H. T. Phys. Rev. B: Condens. Matter Mater. Phys. 2011, 84, 155303.

(57) Miller, D. A. B.; Chemla, D. S.; Damen, T. C.; Gossard, A. C.; Wiegmann, W.; Wood, T. H.; Burrus, C. A. Phys. Rev. Lett. 1984, 53 (22), 2173-2176. 